\title{
THE ORDER OF ENTIRE FUNCTIONS WITH RADIALLY DISTRIBUTED ZEROS
}

\author{
FARUK F. ABI-KHUZAM
}

\begin{abstract}
It is shown that an entire function with radially distributed zeros has finite order $\lambda$ if it has finite lower order $\mu$. It is then shown that functions with real negative zeros only are extremal for the problem of maximizing the Nevanlinna characteristic in the class of entire functions satisfying $\lambda-\mu>1$.
\end{abstract}

Let $\lambda, \mu, \rho$ be the order, lower order and the exponent of convergence of the zeros of an entire function $f$. Whittaker [8, p. 130] has shown that if $\mu$ and $\rho$ are finite, then $\lambda$ is finite and $\lambda=\max (\mu, \rho)$. The finiteness of $\mu$ by itself, however, is not enough to make $\lambda$ finite. It is a rather interesting fact, that a radial distribution of the zeros of $f$ makes $\lambda$ finite if $\mu$ is finite. We point out that the theorem whose statement constitutes the title of Whittaker's paper [8], is an immediate corollary of earlier and more informative results of Edrei and Fuchs [2, p. 298], [3, pp. 261, 264].

Using rather difficult estimates of $T(r, f)$, Edrei and Fuchs [2, p. 308] have shown that $q \leqslant \mu$ for a canonical product $f$ of finite genus $q(>1)$ having only real negative zeros. Their result implies that $q<\mu<\lambda<q+1$ for such functions provided that $\lambda$ is assumed finite. Years later Shea [6, p. 204], in studying the Valiron deficiencies of meromorphic functions, obtained as a corollary a bound on $\lambda$ in terms of $\mu$ only, for entire functions $f$ having only real negative zeros and finite order $\lambda$.

Our first result (Theorem 1 below) generalizes the above results and the proof extends to subharmonic (and $\delta$-subharmonic) functions in space. In addition, our proof may be of interest because of its simplicity.

THEOREM 1. Let $f$ be an entire function of order $\lambda$ and lower order $\mu$. Assume that all the zeros of $f$ lie on the radii defined by

$$
r e^{i \omega_{0}}, r e^{i \omega_{1}}, \ldots, r e^{i \omega_{m}} \quad(r>0, m>0),
$$

where the $\omega$ 's are real.

Then $\lambda$ is finite if and only if $\mu$ is finite.

If $m=0$ and $\mu$ is finite then $\lambda<[\mu]+1$.

Entire functions whose zeros lie on a ray are believed to be extremal for a large class of problems in Nevanlinna theory. Let $f$ be entire with zeros $\left\{a_{n}\right\}$ and nonintegral order $\lambda$, and let $F$ be the canonical product with zeros $\left\{-\left|a_{n}\right|\right\}$. If

Received by the editors April 22, 1979 and, in revised form, March 31, 1980.

AMS (MOS) subject classifications (1970). Primary 30A64, 31A10; Secondary 30A68.

Key words and phrases. Order, lower order of an entire function. 
$0<\lambda<1$, then it is a consequence of Gol'dberg's lemma [4, p. 106] that $T(r, f)<$ $T(r, F)$, but nothing is known if $\lambda>1$. In this direction the following result may be of interest.

THEOREM 2. Let $f$ be entire of finite nonintegral order $\lambda$ and lower order $\mu$. If $\lambda-\mu>1$, then there exists a sequence $\left\{x_{n}\right\}$ increasing to infinity and a positive $\gamma$ $(<1)$ such that

$$
T(r, f)<T(r, F), \quad x_{n}^{\gamma}<r<x_{n} .
$$

Connected to our Theorem 1 is the following unpublished result of I-Lok Chang.

THEOREM A. Let $f$ be entire $f(0)=1$, and let $\left\{a_{j}\right\}$ be the sequence of its zeros. Take $N(r, 1 / f)$ to be the counting function that appears in Nevanlinna's theory.

Let $k \geqslant 1$ be an integer and let

$$
\sum_{j=1}^{\infty}\left|a_{j}\right|^{-k}=+\infty .
$$

Consider the point-set

$$
\Delta_{k}=\left\{z:\left|\arg z^{k}\right|<\beta<\pi / 2\right\}
$$

and let

$$
\sum_{a_{j} \notin \Delta_{k}}\left|a_{j}\right|^{-k}<+\infty
$$

Then, Nevanlinna's characteristic $T(r, f)$ satisfies the relation

$$
T(r, f)>\frac{1}{2} N(r, 1 / f)+r^{-k} \Omega(r)
$$

with $\Omega(r) \rightarrow+\infty$ as $r \rightarrow+\infty$.

Since $r^{-k} T(r, f)$ always tend to a limit (possibly infinite) when $\sum_{j=1}^{\infty}\left|a_{j}\right|^{-k}<+\infty$, we may 'append' the obvious corollary of Theorem $A$ to obtain

THEOREM B. If $f$ is an entire function satisfying (3) and (4) for some positive integer $k$, and if $T(r, f)$ is its Nevanlinna characteristic, then $\lim _{r \rightarrow \infty} r^{-k} T(r, f)$ exists as a finite or infinite limit.

The next corollary shows the connection between Chang's result and Theorem 1 .

Corollary of Chang's TheOrem. Let $f$ be an entire function having all its zeros on the two rays

$$
r, r e^{i \pi m / \alpha} \quad(r>0, \alpha(>1), m \text { integers }) .
$$

If the lower order $\mu$ of $f$ is finite, then its order $\lambda$ is finite and $\lambda<[\mu]+2 \alpha$.

ProOF OF COROLlary. Let $f$ satisfy the conditions of the corollary and suppose first that $m$ is even and has no common factors with $\alpha$. Let $k$ be the (unique) multiple of $\alpha$ in the set $[\mu]+1,[\mu]+2, \ldots,[\mu]+\alpha$. Then all the zeros of $f$ lie in $\Delta_{k}$ and condition (4) of Theorem A is satisfied. By Theorem B the $\lim _{r \rightarrow \infty} r^{-k} T(r, f)$ exists. Since $k>\mu$, this limit must be finite. It follows that $\lambda<k$ and so $\lambda<k<$ $[\mu]+\alpha<[\mu]+2 \alpha$. The case when $m$ is odd may be proved similarly, but $k$ must 
be taken to be a multiple of $2 \alpha$. We remark that examples of Edrei and Fuchs [2, p. $295]$ show that the bound $[\mu]+\alpha$, obtained when $m$ is even, is sharp. We also note that, if instead of one ray, we have a finite number of rays of arguments $m_{1} \pi / \alpha_{1}$, $m_{2} \pi / \alpha_{2}, \ldots, m_{s} \pi / \alpha_{s}$, then a function having all its zeros on these rays and having lower order $\mu$ will have order $\lambda$ bounded above by $[\mu]+2$ (lowest common multiple of $\alpha_{1}, \alpha_{2}, \ldots, \alpha_{s}$ ). We finally point out that the corollary may be proved directly from Theorem A.

Proof of Theorem 2. Let $f$ be of finite nonintegral order $\lambda$, then $N(r)=$ $N(r, 1 / f)$ has order $\lambda$. Then $F$ has order $\lambda$. By Theorem 1, the lower order $\mu^{\prime}$ of $F$ satisfies $\lambda-\mu^{\prime} \leqslant 1$ and so $\mu<\mu^{\prime}$. Choose $\varepsilon(>0)$ so that $\mu<\mu^{\prime}-\varepsilon$ and then choose $\gamma$ such that $\mu /\left(\mu^{\prime}-\varepsilon\right)<\gamma<1$. By Whittaker's Lemma [8, p. 130] there exists a sequence $\left\{x_{n}\right\}$ increasing to infinity such that

$$
T(r, f) \leqslant r^{\mu^{\prime}-\varepsilon} \quad\left(x_{n}^{\gamma}<r<x_{n}\right) .
$$

Since $T(r, F) \geqslant r^{\mu^{\prime}-\varepsilon}$ for all large $r$, (1) follows.

Proof of Theorem 1. Let $f$ be an entire function satisfying the conditions of Theorem 1 and assume that its lower order $\mu$ is finite. We first show that the condition of the theorem implies that the zeros of $f$ are located in 'suitable' sectors. This we do by following, step by step, an argument of Edrei, Fuchs and Hellerstein [1, p. 149]. Consider the set of arguments $\omega_{j}$ and assume that $\omega_{0}=2 \pi$; this is clearly no restriction. Choose $k(0<k<m)$, and relabel if necessary, so that $\left\{2 \pi, \omega_{1}, \ldots, \omega_{k}\right\}$ is a maximal linearly independent set. If $k<m$, there exists integers $n_{l_{j}}$ and $\sigma(>0)$ such that

Put

$$
\sigma \omega_{l}=2 \pi n_{l_{0}}+\sum_{j=1}^{k} n_{l_{j}} \omega_{j} \quad(l=k+1, \ldots, m) .
$$

$$
M_{l}=\sum_{j=1}^{k}\left|n_{l}\right|, \quad M=\sup \left\{\sigma, M_{k+1}, M_{k+2}, \ldots, M_{m}\right\} .
$$

By Weyl's equidistribution theorem [7], there exists a sequence $\left\{\lambda_{s}\right\}$ of positive integers satisfying

$$
\left|\lambda_{s} \omega_{j}-L_{s j} 2 \pi\right|<\frac{\pi}{(2+\varepsilon) M} \quad(j=1,2, \ldots, k ; s=1, \ldots ; \varepsilon>0),
$$

where the $L_{s j}$ are integers.

Choose $s_{0}$ so that $\sigma \lambda_{s_{0}}>\mu$ and put $q=\sigma \lambda_{s_{0}}$. We are now ready to show that the zeros of $f$ lie in "suitable" sectors: In (9) take $s=s_{0}$, multiply by $\left|n_{l}\right|$ and sum over $j$ from 1 to $k$. In view of (7) and (8) we get

$$
\left|\omega_{l}-\frac{\Delta_{h l} 2 \pi}{q}\right|<\frac{\pi}{(2+\varepsilon) q} \quad(l=k+1, k+2, \ldots, m),
$$

where the $\Delta$ 's are integers.

By (8) and (9), it is clear that (10) holds also for $l=1,2, \ldots, k$, with $\Delta_{h l}=\sigma L_{h l}$. Hence we have

$$
\left|\omega_{l}-\frac{\Delta_{h l} 2 \pi}{q}\right|<\pi /(2+\varepsilon) q \quad\left(l=1,2, \ldots, m ; q>\mu, h=s_{0}\right) .
$$


To continue we write $\log \left|f\left(r e^{i \theta}\right)\right|=\sum_{m=-\infty}^{\infty} c_{m}(r) e^{i m \theta}$. Then we have [5, p. 379]

$$
c_{m}(r)=-\frac{1}{2 m}\left\{\sum_{r<r_{k}<R}\left(r / z_{k}\right)^{m}+\sum_{r_{k}<r}\left(\bar{z}_{k} / r\right)^{m}\right\}+(r / R)^{m} O(T(2 R)),
$$

where $\left\{z_{k}\right\}$ are the zeros of $f$ and $r_{k}=\left|z_{k}\right|$.

In (12) we put $m=q$. Since $q>\mu$, the last term in (12) will tend to zero as $R \rightarrow \infty$ through a suitable sequence $\left\{R_{n}\right\}$. It follows that $\Sigma_{r<r_{k}<R_{k}} z_{k}^{-q}$ tends to a limit as $R\left(=R_{n}\right)$ tends to infinity. If we write $z_{k}=r_{k} e^{i \theta_{k}}$, it follows that $\operatorname{Re}\left\{\Sigma_{r<r_{k}<R_{k}} z_{k}^{-q}\right\}=\Sigma_{r<r_{k}<R} r_{k}^{-q} \cos \left(q \theta_{k}\right)$ tends to a limit as $R\left(=R_{n}\right)$ tends to infinity. Since the arguments $\theta_{k}$ satisfy (11) we have $\cos (q \pi /(2+\varepsilon) q) \sum_{r<r_{k}<R} r_{k}^{-q}$ $<\Sigma_{r<r_{k}<R} r_{k}^{-q} \cos \left(q \theta_{k}\right)$. It follows that $\Sigma_{r<r_{k}<R} r_{k}^{-q}$ is bounded as $R\left(=R_{n}\right)$ tends to infinity, and being an increasing function of $R$, it will have a limit as $R \rightarrow \infty$ unrestricted. Thus $\sum r_{k}^{-q}$ converges and so, the exponent of convergence of the zeros of $f$ is $<q$. By Whittaker's result, $\lambda<\max (\mu, q)$.

When the zeros of $f$ all lie on a ray, we may choose $q=[\mu]+1$. Using this in (22) we obtain $\rho<[\mu]+1$ from which follows that $\lambda<[\mu]+1$. This completes the proof of Theorem 1.

Proof of Theorem B. Let $f$ be an entire function whose zeros satisfy (3) and (4) for some integer $s(>1)$. If $\lim _{\inf _{r \rightarrow \infty}} r^{-s} T(r, f)=\infty$ then $\lim _{r \rightarrow \infty} r^{-s} T(r, f)=\infty$. Suppose then that $\lim _{\inf _{r \rightarrow \infty}} r^{-s} T(r, f)<+\infty$. Then the lower order $\mu$ of $f$ is finite and $\mu \leqslant s$. In (12), take $m=s$ and let $R$ tend to infinity through a sequence $R_{n}$ such that $R_{n}^{-s} T\left(R_{n}, f\right)$ tends to a finite limit. By taking subsequences if necessary and repeating the same arguments after (12), we conclude as before, that $\Sigma\left|a_{j}\right|^{-s}<$ $+\infty$. It follows that $f$ is of finite order $\lambda<s$. Thus we may write $f(z)=e^{Q(z)} P(z)$ where $Q$ is a polynomial of degree $d<s$ and $P$ is a Weierstrass product of genus $s-1$. Since for such products $P$, even when not canonical, $T(r, P)=o\left(r^{s}\right)$ as $r \rightarrow \infty$, we conclude by the elements of the theory that $\lim _{r \rightarrow \infty} r^{-s} T(r, f)$ exists and is $<+\infty$. This completes the proof of Theorem B.

REMARK. The possibility that $T(r, f)<T(r, F)$ for a set that contains arbitrarily large values of $r$ is further supported by the following: Let

$$
f(z)=e^{p(z)} \prod_{n=1}^{\infty} e\left(z / z_{n}, q\right) \text { and } F(z)=e^{p(z)} \prod_{n=1}^{\infty} E\left(z /-\left|z_{n}\right|, q\right)
$$

be two entire functions, with $p(z)=a_{0}+a_{1} z+\cdots+a_{q} z^{q}$, and $P(z)=\left|a_{0}\right|$ $+\cdots+\left|a_{q}\right| z^{q}$ and $q=$ the greatest integer less than or equal to the order $\lambda$ of $f$ which we assume finite. Then we have [5, p. 380]

$$
\left|c_{m}(r ; f)\right|<\left|c_{m}(r ; F)\right|<2 T(r, F)-N\left(r, \frac{1}{F}\right), \text { for all } m \text {. }
$$

In the proof of the approximation lemma of Edrei and Fuchs [2, p. 312] we apply inequality (13) in place of their inequality (8.8). The result is that in the error term appearing in their lemma, we may replace $T(r, f)$ by $T(r, F)$.

In ending this paper I wish to thank the referee for pointing out the existence of Chang's result and its connection to Theorem 1 . 


\section{REFERENCES}

1. A. Edrei, W. H. J. Fuchs and S. Hellerstein, Radial distribution and deficiencies of the values of a meromorphic function, Pacific J. Math. 11, (1961), 135-151.

2. A. Edrei and W. H. J. Fuchs, On the growth of meromorphic functions with several deficient values, Trans. Amer. Math. Soc. 93 (1959), 292-328.

3. , Valeurs deficients et valeurs asymptotiques des fonctions meromorphes, Comment. Math. Helv. 34 (1960), 258-295.

4. W. K. Hayman, Meromorphic functions, Pergamon Press, Oxford, 1964.

5. J. Miles and D. F. Shea, An extremal problem in value-distribution theory, Quart. J. Math. 24 (95) (1973), 377-383.

6. D. F. Shea, On the Valiron deficiencies of meromorphic functions of finite order, Trans. Amer. Math. Soc. 124 (1966), 20-27.

7. H. Weyl, Uber die Gleichverteilung von Zahlen mod. I, Math. Ann. 77 (1916), 313-352.

8. J. M. Whittaker, Entire functions of irregular growth take every value, Bull. London Math. Soc. 4 (1972), 130-132.

Department of Mathematics, American University of Beirut, Beirut, Lebanon

Current address: Department of Mathematics, Syracuse University, Syracuse, New York 13210 